\title{
Numerical Simulation OF NoN-Evaporating AND NON-REACTING HEAVY FUEL OIL INJECTION SPRAY IN MEDIUM SPEED ENGINES USING OPENFOAM
}

\author{
Hashem Nowruzi ${ }^{1}$, Parviz Ghadimi ${ }^{2 *}$, Mehdi Yousefifard ${ }^{3}$, Mohammad A. Feiz \\ Chekab $^{4}$
}

1,2*,3,4 Department of Marine Technology, Amirkabir University of Technology, Tehran, Iran

\begin{abstract}
In the current study, Computational Fluid Dynamics (CFD) is utilized to investigate the non-evaporating and non-reacting spray of Heavy Fuel Oil (HFO) in medium speed engine at three different high pressure injections. An Eulerian-Lagrangian multiphase scheme is used to simulate C14H30 as heavy fuel oil spray. OpenFOAM freeware is modified and implemented to study the effect of injection pressure on the spray characteristics. HFO is injected into a constant volume chamber under specified injection parameters. The obtained numerical results are compared against existing experimental data and good compliance is achieved, especially at lower injection pressure. Moreover, CFD results show that higher injection pressure enhances the atomization, while increases the average velocity at timeline in the Z-direction. Consequently, particle diameter decreases, number of particles increases and spary efficiency improves. On the other hand, change in injection pressure from 600 to 1000 bar is more apparent than change from 1000 bar to 1400 bar on the basic parameters in spray analysis.
\end{abstract}

\section{KEYWORDS}

Diesel Spray; OpenFOA; Heavy Fuel Oil; High Injection Pressure; Medium Speed Diesel Engine

\section{INTRODUCTION}

Medium and low speed marine diesel engines are immensely important because large part of world ocean fleet use medium and low marine diesel engines. Accordingly, researchers in the field of diesel heavy industry are seeking different solutions to improve the performance and efficiency of marine diesel engines. In this regard, the role of increasing the injection pressure has been more critical in recent years. High pressure injection leads to an increase in the wellatomized fuel spray and more oxygen availability because of enhanced fuel-air mixing.

Many experiments have thus far been carried out to investigate the effects of high pressure injection on spray characteristics. However, because of complexity of this task, the development of Computational Fluid Dynamics (CFD) methods has become an alternative for conducting experimental research in engine. Accordingly, different numerical codes and commercial softwares such as KIVA, STAR-CD and AVL FIRE have been developed for diesel engines. On the other hand, the development of open source CFD softwares in the field of diesel engines has been considered an important goal. One of the most popular open source CFD software is OpenFOAM. Gjesing et al. [1] presented coupled atomization and spray numerical model in spray forming with focus on the coupling effect of the melt break-up phenomena using OpenFOAM. 
On the other hand, Kassem et al. [2] Implemented eddy dissipation model in OpenFOAM for non-premixed Methane jet flame.

Spray fundamental parameters such as spray penetration length, number of particle and their diameter, average particle velocity and spray structure has essential role in improving the performance and efficiency of marine diesel engines. In addition, the main fuel for large marine Diesel engines is Heavy Fuel Oil (HFO). This fuel is mainly a combination of hydrocarbons of metallic and non-metallic fraction. Several studies dealing with the investigation of spray and combustion modeling in marine diesel engine and usage of heavy fuel oil have been reported. Goldsworthy [3] presented a simplified numerical model for vaporization and combustion of heavy residual based fuel oil at high-pressure sprays in marine diesel engines. Fink et al. [4] reported experimentally investigation of HFO, Fuel Water Emulsion and diesel fuel effects on spray parameter in medium speed engine for marine application. Kyriakides et al. [5] developed and implemented a model with the thermophysical properties of Heavy Fuel Oil for marine diesel engine using KIVA CFD code. Their simulations have been performed for two values of chamber pressure at a constant-volume for investigation of the effect of fuel properties on spray atomization. Struckmeier et al. [6] numerically developed evaporation, ignition and combustion models for a two-component fuel for KIVA CFD code. Chryssakis et al. [7] presented a test of HFO model for both a visual constant-volume combustion chamber and a fuel ignition analyzer for large marine diesel engine. A test facility to realize condition relevant to the combustion system present in large two stroke marine diesel engines has been designed by Herrmann et al. [8] and comprehensive simulation have been performed for appropriate layout and dimensioning of the fundamental component.

Herrmann et al. [9] also experimentally presented a novel setup for study of spray and combustion processes at conditions typical of large two stroke marine diesel engines by applying peripheral injection as a swirling flow in a disk shaped constant volume spray combustion chamber. For the same combustion chamber, Herrmann et al [10] presented an investigation of injection and combustion process of HFO as a low fuel quality. Andreadis et al. [11] used KIVA3-based CFD code coupled with an evolutionary algorithm to study the effects of the fuel injection parameters on the performance and emissions formation in a large-bore marine diesel engine. Stamoudis et al. [12] numerically and experimentally investigated an evaporation model for Heavy Fuel Oil (HFO) combustion. In their work, HFO is considered as a mixture of a heavy and a light fuel component, with the thermophysical properties of the heavy component.

In the current work, using open source OpenFOAM software, numerical simulation of nonreacting and non-evaporating injection spray in constant volume chamber of medium speed engine has been conducted. $\mathrm{C} 14 \mathrm{H} 30$ has been considered as heavy fuel oil and simulation has been performed at three different high injection pressures. To accomplish this task, a new Heavy Fuel Oil model along with its thermophysical characteristics is added to OpenFOAM.

\section{TECHNICAL DESCRIPTION OF THE PROBLEM AND GOVERNING EQUATION}

Fluids behavior can be modeled by five different equations including conservation of mass, energy, and the vectorial components of the momentum. Additionally, in the case of multiphase flow, a set of models to account for the breakup and mass transfer as source terms must be considered. Also, Lagrangian Particle Tracking (LPT) approach is employed for liquid droplet modeling in this paper. Conservation equation of mass can be described by (1):

$$
\frac{\partial \rho}{\partial t}+\frac{\partial \rho u_{j}}{\partial x_{j}}=S_{e v},
$$


where $S_{e v}$ represents the contribution of evaporation of fuel as a source term. Momentum conservation is obtained as in

$$
\frac{\partial \rho u_{i}}{\partial t}+\frac{\partial}{\partial x_{j}}\left(\rho u_{i} u_{j}-\tau_{i j}\right)=-\frac{\partial p}{\partial x_{i}}+S_{i, m o},
$$

Here, source term due to interaction with the spray liquid phase is included in $\mathrm{S}_{\mathrm{i}, \mathrm{mo}}$. In addition, energy conservation is proportional is given in Eq.3.

$$
\frac{\partial \rho h}{\partial t}+\frac{\partial}{\partial x_{j}}\left(\rho u_{j} h-\frac{\rho v}{\operatorname{Pr}} \frac{\partial h}{\partial x_{j}}\right)=\frac{\partial p}{\partial t}+\frac{\partial p u_{j}}{\partial x_{j}}+S_{h e},
$$

Here, $S_{h e}$ is a source term due to the heat that is transfered from the liquid phase and $\operatorname{Pr}$ represents the Prandtl number. Species conservation equation, based on this concept that a gas phase mainly contains air and vaporized fuel, is presented. Hence, in order to measure the mass fraction of fuel ( $X_{f}$ ) for each point of the gas phase, Eq.4 is utilized.

$$
\frac{\partial \rho X_{f}}{\partial t}+\frac{\partial}{\partial x_{j}}\left(\rho u_{j} X_{f}-\rho D \frac{\partial X_{f}}{\partial x_{j}}\right)=S_{e v},
$$

For considering lagrangian particle tracking scheme, it should be noted that the liquid phase is formed by disperse droplets of fuel. Therefore, a stochastic approach in which droplets are considered discrete entities is feasible. A probability conservation equation can be derived from Eq.5.

$$
\frac{\partial f}{\partial t}+\nabla_{x} \cdot(f u)+\nabla_{v} \cdot\left(f \frac{\partial u}{\partial t}\right)+\frac{\partial}{\partial r}\left(f \frac{\partial r}{\partial t}\right)+\frac{\partial}{\partial T}\left(f \frac{\partial T}{\partial t}\right)+\frac{\partial}{\partial y}\left(f \frac{\partial y}{\partial t}\right)+\frac{\partial}{\partial \dot{y}}\left(f \frac{\partial \dot{y}}{\partial t}\right)=f_{c o}+f_{b r}
$$

Here, $f\left(x, u, t, r, T, y, y^{\prime}\right)$ is a probable number of droplets per unit volume at a given position ( $\mathrm{x}$ ), given time ( $\mathrm{t}$ ), radius ranging from $r$ to $r+d r$, temperature and velocity respectively from $T$ to $T+d T$ and from $u$ to $u+d u$ with droplet distortion parameters from $y$ to $y+d y$ and until $\dot{\mathrm{y}}+\mathrm{d} \dot{\mathrm{y}}$. There are also two source parameters of $\left(f_{c o}\right)$ and $\left(f_{b r}\right)$ that indicate the effects of collision and breakup of the droplets, respectively.

The breakup modeling used in this simulation is ReitzDiwakar model that was presented by Reitz and Diwakar [13]. Furthermore, the applied turbulence model for simulation of the gas field, is $\mathrm{K}-\mathrm{o}$.

\section{NUMERICAL MODEL}

For numerical simulation of the fuel characteristics, the Stamoudis et al. [12] HFO model has been added to OpenFOAM. In this study, C14H30 thermophysical properties as a HFO generator are implemented in Fuel Library of OpenFOAM. For this purpose, the NASA jannaf coefficient 
for thermophysical properties of $\mathrm{C} 14 \mathrm{H} 30$ is calculated and applied to thermophysical properties. Fundamental characteristic of the modeled Heavy Fuel Oil are presented in Table 1.

Table 1. Considered heavy fuel oil characteristics.

\begin{tabular}{|l|l|l|}
\hline Parameter & Value & unit \\
\hline Chemical formulation & $\mathrm{C} 14 \mathrm{H} 30$ & --- \\
\hline Viscosity & 12 & $\frac{\mathrm{mm}^{2}}{\mathrm{~s}}$ \\
\hline Density & 895 & $\frac{\mathrm{Kg}}{\mathrm{m}^{3}}$ \\
\hline Activation energy (E) & 77.4 & $\frac{\mathrm{KJ}}{\mathrm{mol}}$ \\
\hline
\end{tabular}

Physical characteristics of the modeled heavy fuel, and in particular the particularly viscosity, have a key role on the structure of spray formation and its fundamental properties. It must also be noted that these fuel physical characteristics are temperature dependent. On the other hand, the Fink et al. [4] chamber and injection parameter has been applied to OpenFOAM. The model set up for combustion chamber parameter and injection parameters can be seen in Table 2.

Table 2. Model set up for combustion chamber parameter and injection parameters.

\begin{tabular}{|l|l|l|l|}
\hline Model Parameter & Value & unit \\
\hline \multirow{4}{*}{$\begin{array}{l}\text { Chamber } \\
\text { parameters }\end{array}$} & Length & 150 & $\mathrm{~mm}$ \\
\cline { 2 - 4 } & Diameter & 50 & $\mathrm{~mm}$ \\
\cline { 2 - 4 } & Pressure & 14 & $\mathrm{bar}$ \\
\cline { 2 - 4 } & Temperature & 298 & $\mathrm{~K}$ \\
\hline \multirow{4}{*}{$\begin{array}{l}\text { Injection } \\
\text { parameters }\end{array}$} & Nozzle Diameter & 0.27 & $\mathrm{~mm}$ \\
\cline { 2 - 4 } & Fuel Injection Pressure & $600-1000-1400$ & $\mathrm{bar}$ \\
\cline { 2 - 4 } & Initial Temp. of Fuel in Injection & 375 & $\mathrm{~K}$ \\
\cline { 2 - 4 } & Injection total Mass & 34 & $\mathrm{mg}$ \\
\hline
\end{tabular}

Based on table 2, it can be seen that volume of the chamber is constant during the spray forming. Also, high injection pressure with equal incremental steps has been considered for investigating the effect of high injection pressure increase. Initial temperature of fuel in injection indicates the injection spray modeling at room temperature.

Lagrange injection droplet size used as boundary conditions at the nozzle is the same as injector's nozzle hole size which is equal to $0.27 \mathrm{~mm}$. Atomization region is the first region in fuel spray process after the entry of liquid fuel into combustion chamber from the injector's nozzle. Adjacent portion of nozzle in the atomization region is a liquid fuel core that has the same size as the injector nozzle. Therefore, liquid fuel core as a Lagrange droplet for boundary condition at the nozzle is considered to have the injector's nozzle hole size. Furthermore, liquid fuel core is decomposed into blobs, ligaments and droplet due to the break-up phenomenon in the atomization region. Second region after atomization region is dense spray region. In dense spray region, blobs disintegrate to ligaments and the ligaments decompose to droplets. Subsequently, in the dilute region as the ending region of fuel spray structure, ligaments decompose to droplets. Therefore, numerous droplets are the main characteristics of the dilute area. 
Next, validation and the obtained results from modeling are presented.

\section{RESULTS AND DISCUSSIONS}

Increase in injection pressure is an important influential factor on spray length and structure of the spray. Time history of spray formation for the injection pressure of 600 bar can be seen in Fig.1.
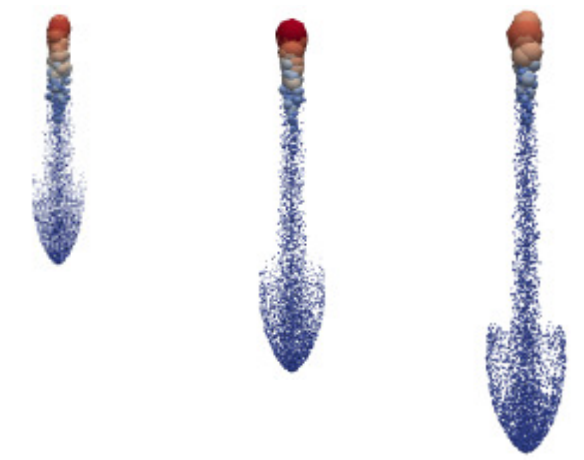

$0.5 \mathrm{~ms} \quad 1 \mathrm{~ms} \quad 1.5 \mathrm{~ms}$

Fig.1. Time history of spray formation at injection pressure of 600 bar.

As evidenced in Fig.1, when spray penetration length increases over time, the tip of spray fuel jet expands in combustion chamber, as well.

Time history of spray formation for the injection pressure of 1000 bar and 1400 bar are presented in Figs. 2 and 3, respectively. Based on these results, it can be concluded that with an increase in injection pressure, the growth rate of spray length in timeline rises.
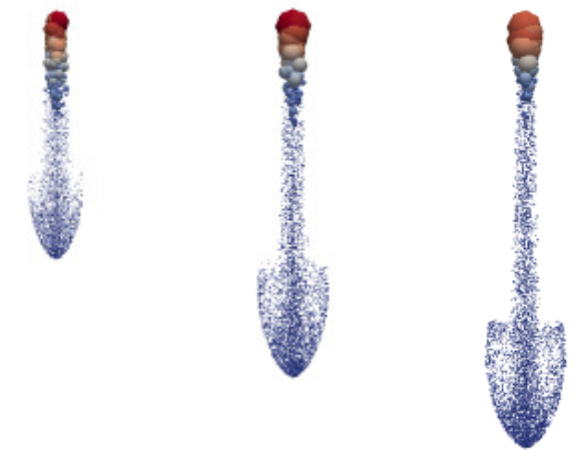

$$
0.5 \mathrm{~ms} \quad 1 \mathrm{~ms} \quad 1.5 \mathrm{~ms}
$$

Fig.2. Time history of spray formation at injection pressure of 1000 bar. 
International Journal of Recent advances in Mechanical Engineering (IJMECH) Vol.3, No.2, May 2014
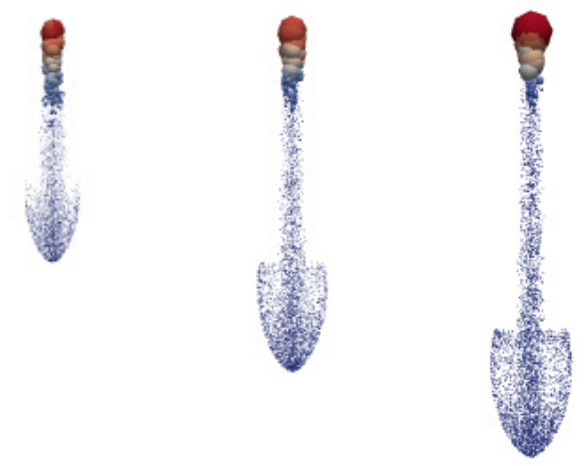

\section{$0.5 \mathrm{~ms} \quad 1 \mathrm{~ms} \quad 1.5 \mathrm{~ms}$}

Fig.3. Time history of spray formation at injection pressure of 1400 bar.

The results of spray length as a validation case for injection pressure of $600 \mathrm{bar}, 1000 \mathrm{bar}$ and 1400 bar in comparison with experimental study conducted by Fink et al. [4] are presented in Figs.4, 5 and 6.
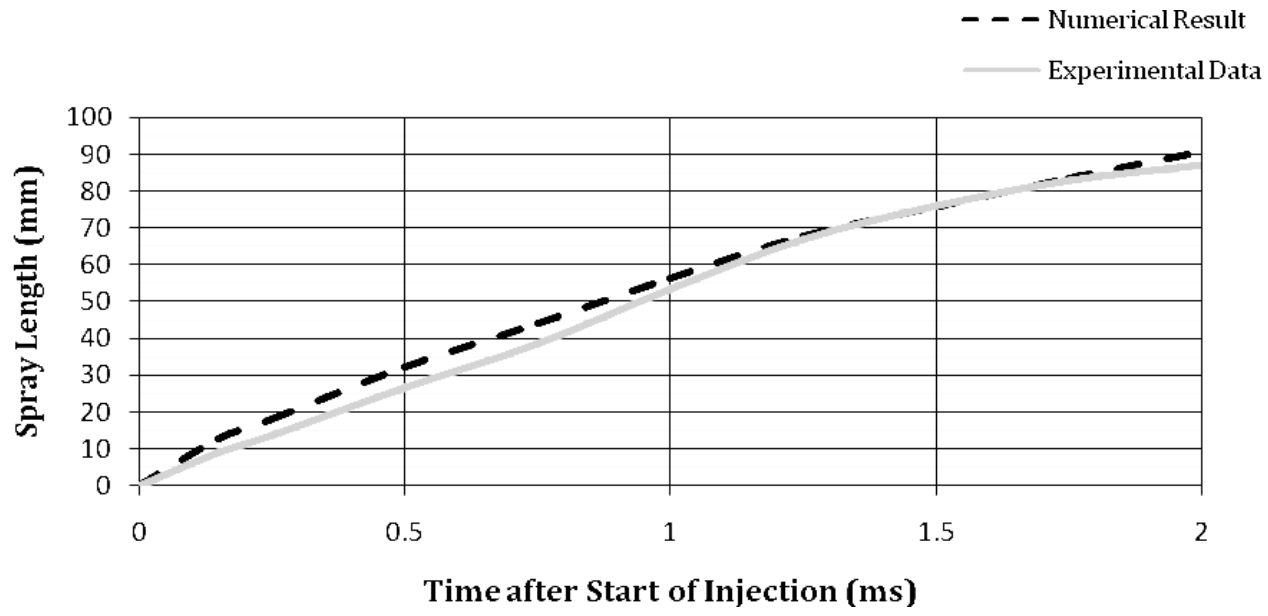

Fig.4. Comparison of numerical and experimental spray length at injection pressure of 600 bar [4].

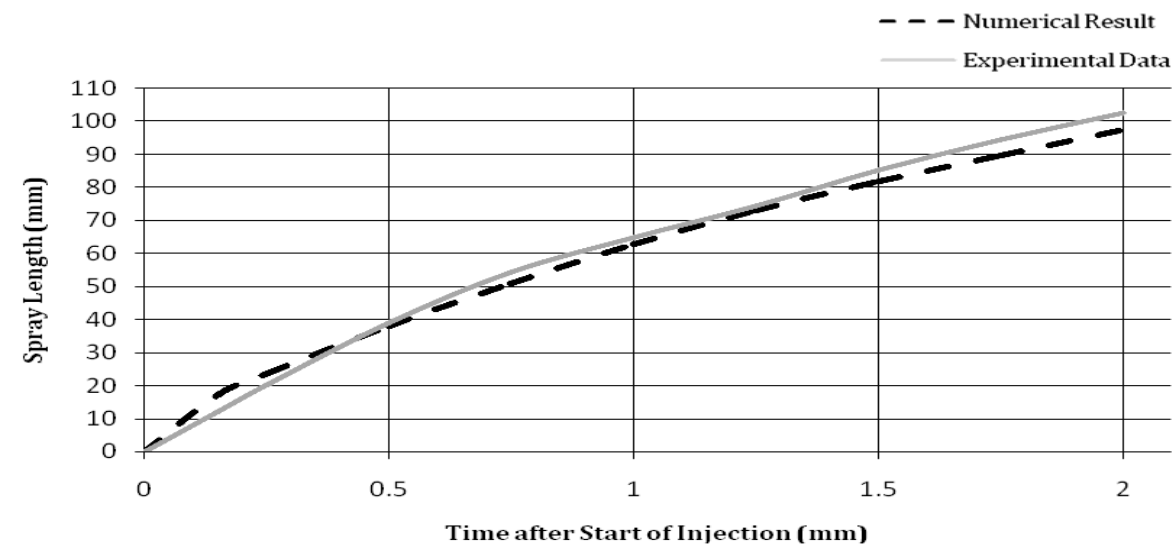

Fig.5. Comparison of numerical and experimental spray length at injection pressure of 1000 bar [4]. 


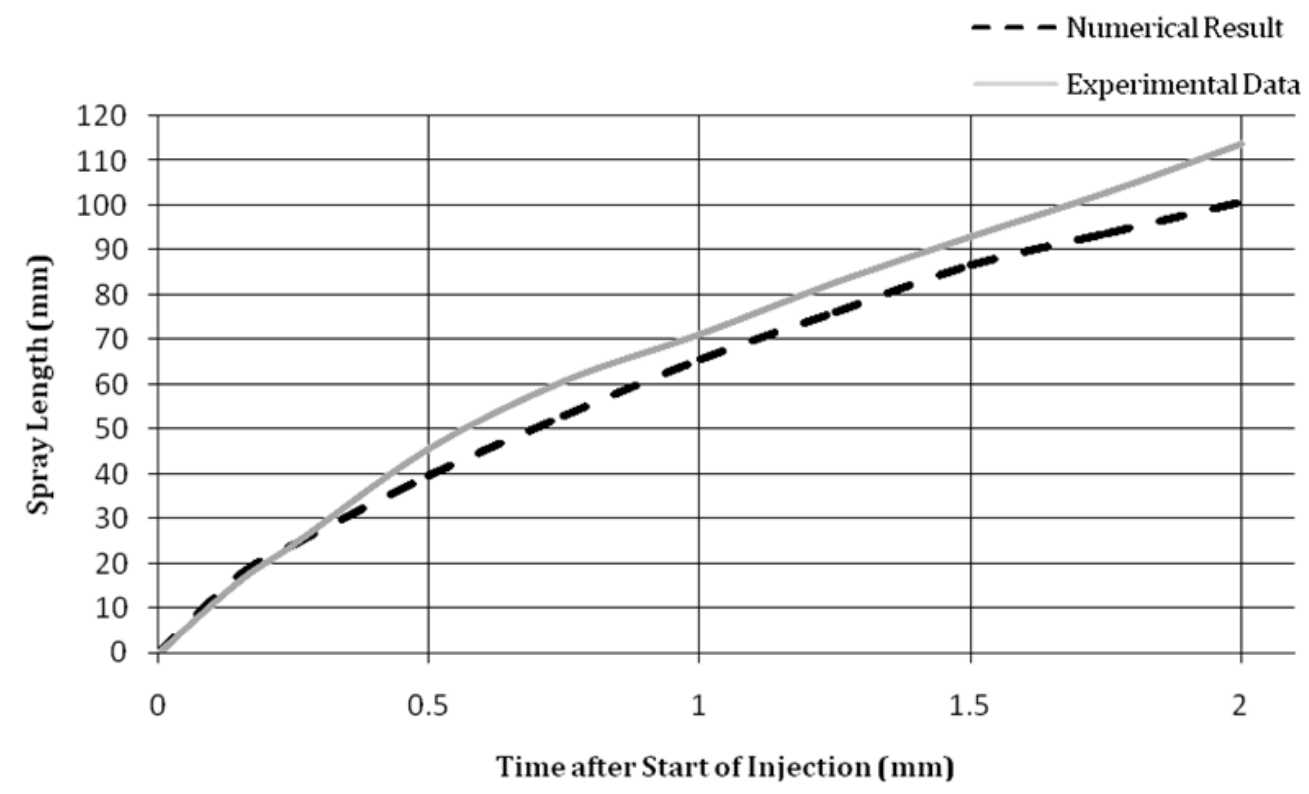

Fig.6. Comparison of numerical and experimental spray length at injection pressure of 1400 bar [4].

Analyses of the results in Figs.4, 5 and 6 indicate acceptable conformity between the numerical findings of OpenFOAM and the experimental results. The computed root mean square of error (RMSE) of experimental and numerical results in pressure injection of 600 bar is 3.34 while the RMSE of the injection pressure of 1000 bar and 1400 bar are 3.36 and 6.85, respectively. It can therefore be concluded that, for lower injection pressure, there is a greater compliance.

In the meantime, what can be seen from the phenomenological perspective of spray length is that, when the pressure rises, the spray length is increased. This increase in injection pressure from 600 bar to 1000 bar in comparison to an increase from 1000 bar to 1400 bar is more evident.

Number of fuel particles, range of particle diameter in parcel and range of parcel velocity in Zdirection as a non-reactive injection spray analysis criteria on computational plane located at 40 $\mathrm{mm}$ from the injector nozzle position in Z-direction have also been studied (as shown in Fig.7).

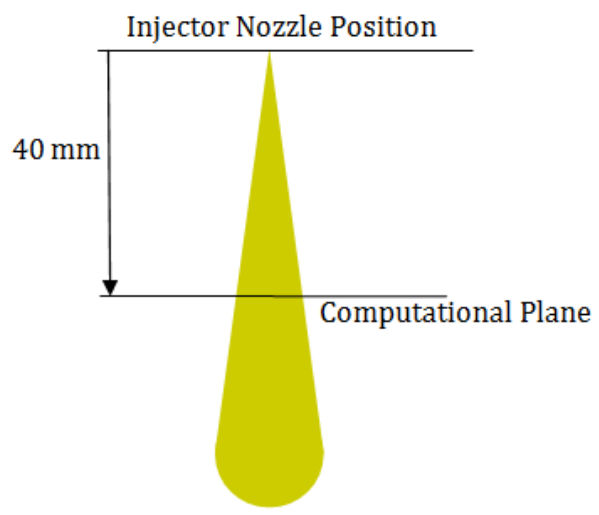

Fig.7. Computational plane located at $40 \mathrm{~mm}$ from the Injector Nozzle Position in Z-direction. 
The computed value of the number of fuel particles on horizontal plane at $40 \mathrm{~mm}$ from the injector at different injection pressures can be seen in Fig.8. By comparing the number of particles at different injection pressures, it is quite evident that the number of particle increases with an increase in the pressure. On the other hand, it can be concluded that higher breakup in atomization phenomenon occurs due to higher pressure. Also, the fluctuation observed in the number of particles in Fig. 8 is attributed to the changes of volume and mass of the moving spray in the computational plane at $40 \mathrm{~mm}$ from the injector.

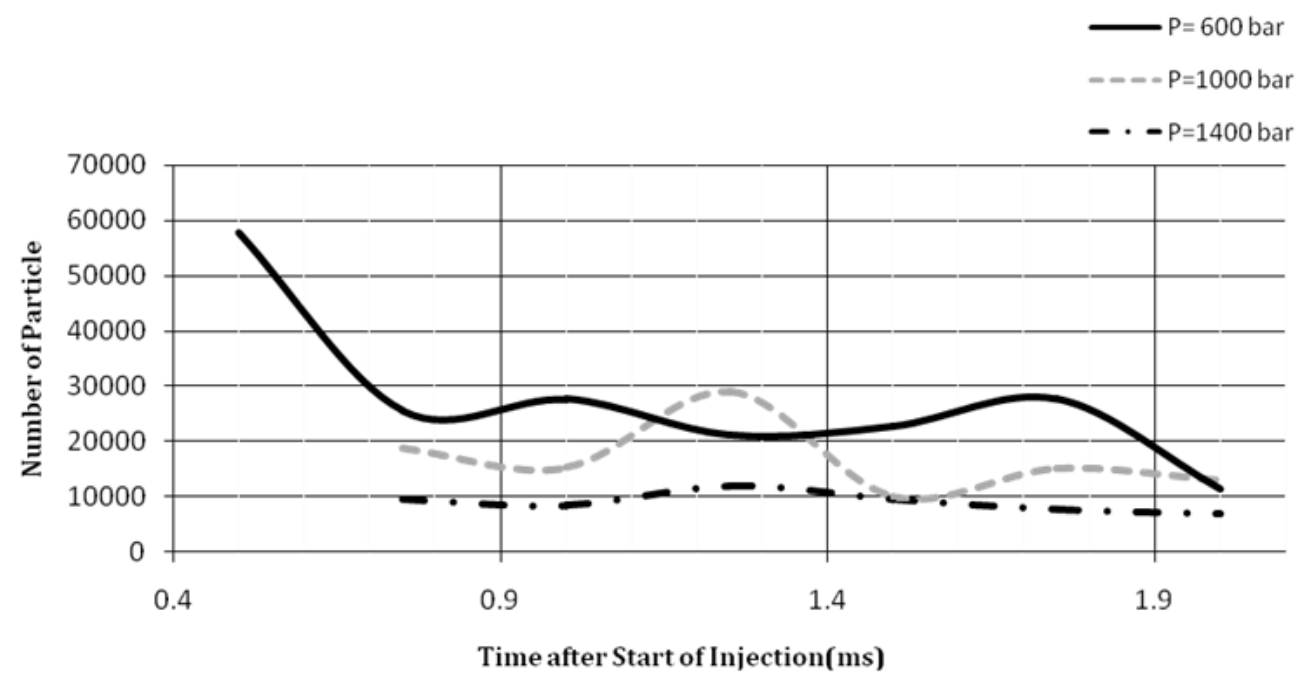

Fig.8. Number of fuel particles on horizontal plane at 40mm from the injector at different injection pressures.

Range of particle diameter in parcel on horizontal plane at $40 \mathrm{~mm}$ from the injector and at different injection pressures are presented in Figs.9, 10 and 11. Based on these results, it is observed that an increase in injection pressure causes a decrease in particle diameter in parcel on horizontal plane at $40 \mathrm{~mm}$ from the injector. This observation is consistent with the fact that increase in the number of particles is due to the enhancement of atomization rate, which itself the result of an increase in injection pressure. On the other hand, a decrease in particle diameter which is due to an increase in fuel injection pressure causes the heavy fuel oil spray to vaporize quickly. However, as a result of a decrease in fuel particles, their inertia will decrease and consequently fuel cannot penetrate deeply into the combustion chamber.

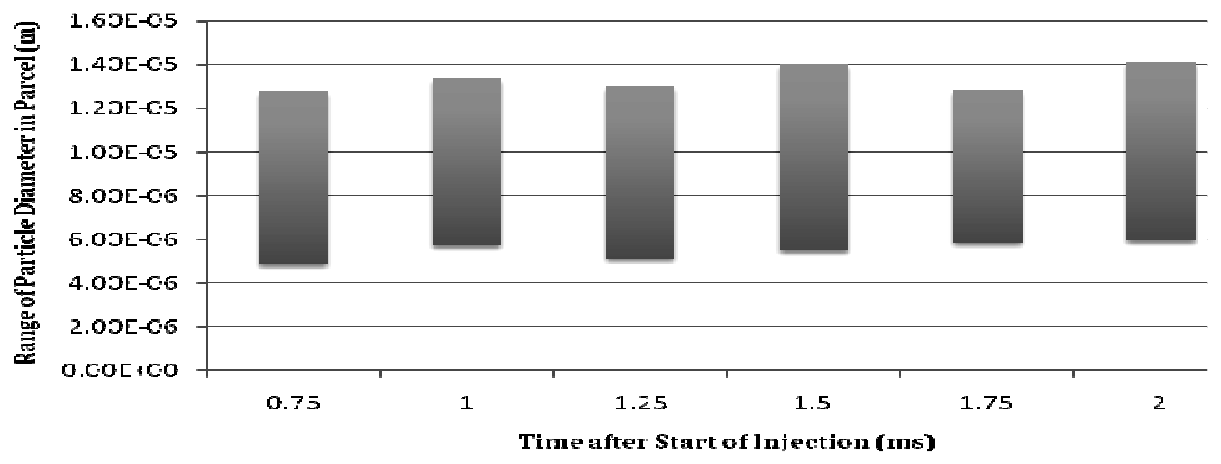

Fig.9. Range of particle diameter in parcel on horizontal plane at 40mm from injector at injection pressure of 600 bar. 


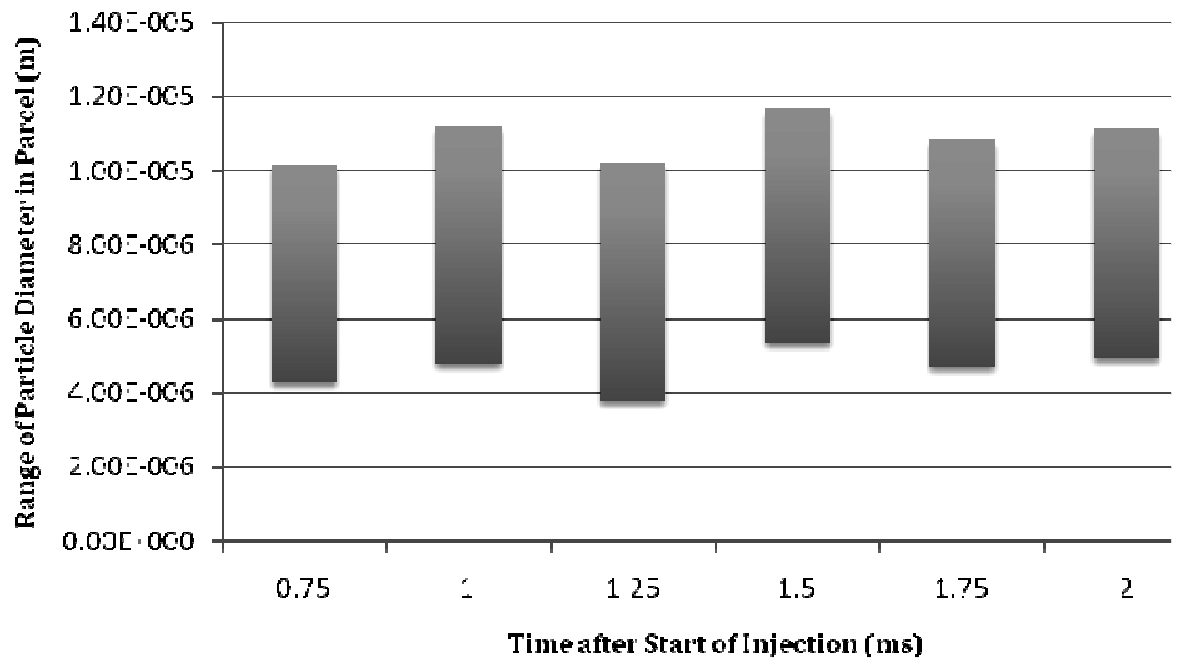

Fig.10. Range of particle diameter in parcel on horizontal plane at $40 \mathrm{~mm}$ from injector at injection pressure of 1000 bar.

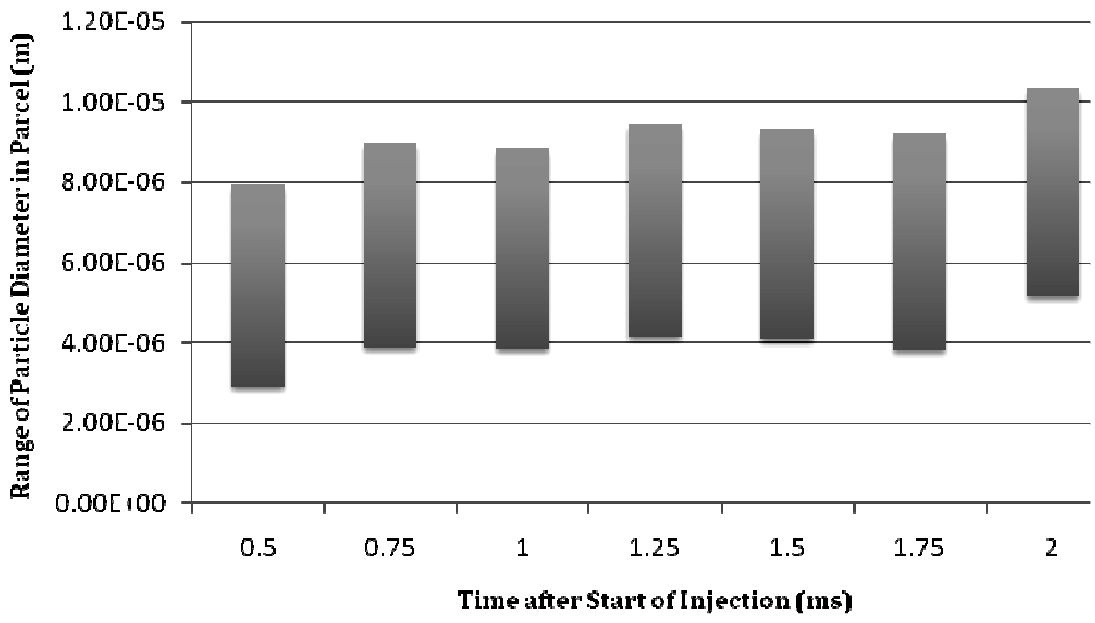

Fig.11. Range of Particle Diameter in Parcel on Horizontal Plane at 40mm from Injector at Injection Pressure of 1400 bar.

Average parcel velocity in Z-direction on horizontal plane at $40 \mathrm{~mm}$ from injector at different injection pressures is presented in Fig.12. Comparison of plots of different injection pressures in Fig.12 indicates that the general average velocity in timeline in Z-direction has increases as the injection pressure increases. The effect of injection pressure change from 600 bar to 1000 bar on rising the average parcel velocity in Z-direction is more apparent than injection pressure increasing from 1000 bar to 1400 bar. 


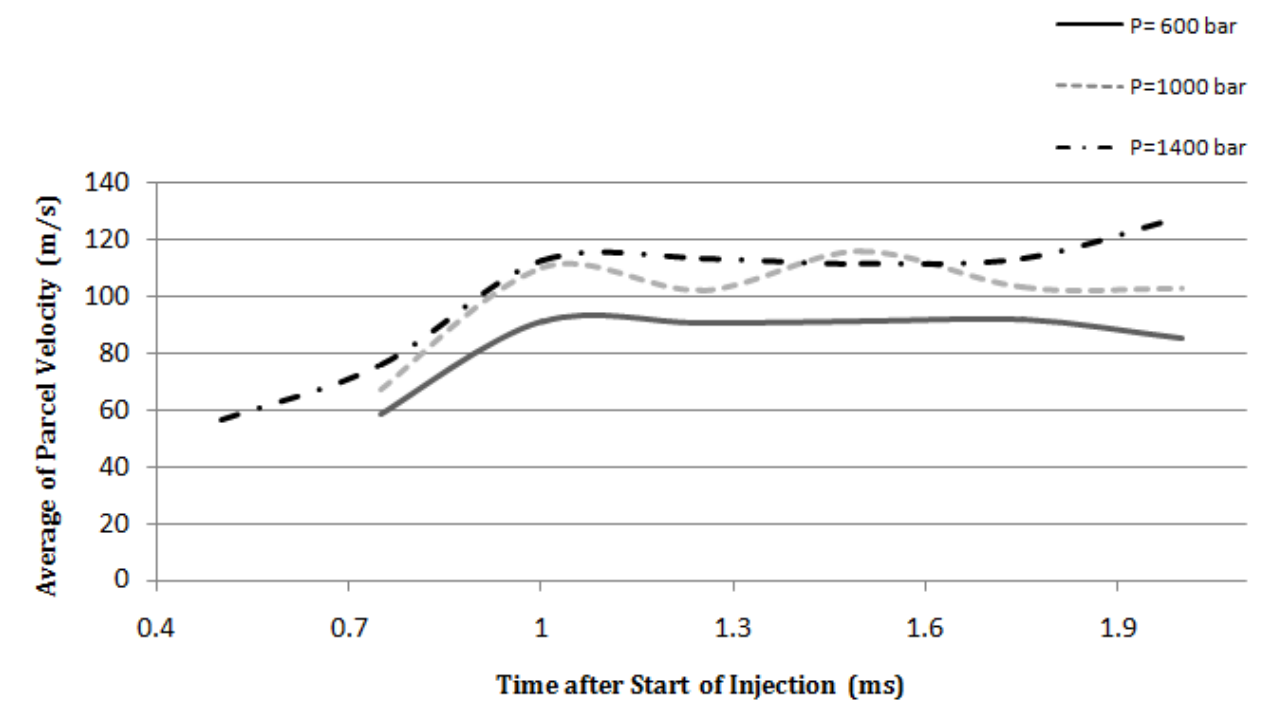

Fig.12. Average parcel velocity in z-direction on horizontal plane at $40 \mathrm{~mm}$ from injector at different injection pressures.

Therefore, it can be concluded from the above results that higher injection pressure improves spray efficiency and performance. On the other hand, more tangible effects on spray efficiency and performance because of injection pressure increasing from 600 to 1000 bar in comparison with injection pressure increasing from 1000 to 1400 bar is observed.

\section{Conclusions}

In this paper, computational fluid dynamic simulations of non-evaporating and non-reacting high pressure spray has been conducted. This was accomplished in a constant volume chamber of medium speed diesel engine using $\mathrm{C} 14 \mathrm{H} 30$ as heavy fuel oil. Modified sprayFoam solver of OpenFOAM freeware was implemented to handle the multiphase flow by Lagrangian Particle Tracking scheme. The breakup model used in this simulation is Reitz-Diwakar model. In addition, $\mathrm{K}$-ò turbulence model is employed for this simulation. More importantly, Stamoudis et al. [12] HFO model and Fink et al. [4] chamber and injection parameters have been added to OpenFOAM. Fuel spray lengths, number of particles, range of particle diameter in parcel, average parcel velocity in Z-direction and structure of the spray have been presented at three different high injection pressures of 600,1000 and 1400 bar.

Fuel library in OpenFOAM has been modified and further developed for applying the thermophysical properties of $\mathrm{C} 14 \mathrm{H} 30$. Main focus of this study has been the assessment of HFO spray structure and fundamental spray parameters influencing the medium speed engine injection efficiency at high injection pressure. The RMSE of the obtained liquied phase spray penetration length against the experimental data at pressure injection of 600 bar is 3.34, while RMSE for pressure injection of 1000 bar and 1400 bar are 3.36 and 6.85, respectively. Based on the computed penetration length, it can be concluded that as the injection pressure increases, the agreement between them decreases. Time history of spray formation shows that, when spray penetration length progresses, the tip of spray fuel jet expands in combustion chamber. The computed spray formation also shows that, an increase in injection pressure would cause a decrease in particle diameter of parcel on the horizontal plane at $40 \mathrm{~mm}$ from the injector. This result is consistent with the expectation that increase in number of particle is due to the 
enhancement of atomization rate, which itseflf results from injection pressure increase. Moreover, general average velocity in timeline in Z-direction also increases as a result of an increase in injection pressure.

Based on the numerical results presented in this paper, higher injection pressure improves spray efficiency and performance, significantly. It is alos quite evident in different charts that this improvement is more apparent in the case of injection pressure increase from 600 bar to 1000 bar. Furthermore, one may also conclude that open source OpenFOAM software is quite capable of simulating the non-reacting HFO injection spray at high pressure, effectively.

\section{REFERENCES}

[1] Gjesing, R., J. Hattel \& U. Fritsching, (2009) "Coupled atomization and spray modeling in the spray forming process using OpenFOAM", Engineering Applications of Computational Fluid Mechanics, Vol. 3.

[2] Kassem, H.I., K.M. Saqr, H.S. Aly \& et al., (2011) "Implementation of the eddy dissipation model of turbulent non-premixed combustion in OpenFOAM", International Communications in Heat and Mass Transfer, Vol. 38.

[3] L., Goldsworthy, (2005) "Computational fluid dynamics modelling of residual fuel oil combustion in the context of marine diesel engines", Int. J. Engine Res. Vol. 7.

[4] C., Fink, B. Buchholz, M. Niendorf \& H. Harndorf, (2008) “ Injection spray analyses from medium speed engines using marine fuels", ILASS.

[5] N., Kyriakides, C. Chryssakis \& L. Kaiktsis, (2009) "Influence of heavy fuel properties on spray atomization for marine diesel engine applications", SAE International.

[6] D., Struckmeier, Tsuru, S. Kawauchi \& H. Tajima, (2009) "Multi-component modeling of evaporation, ignition and combustion processes of heavy residual fuel oil", SAE International Powertrains, Fuels and Lubricants Meeting.

[7] C.,Chryssakis, K. Pantazis \& L. Kaiktsis, (2010) "Combustion modeling with heavy fuel oil forlarge marine diesel engine applications", CIMAC Congress.

[8] K., Herrmann, R. Schulz \& G. Weisser, (2007) "Development of a reference experiment for large diesel engine combustion system optimization", CIMAC Congress.

[9] K., Herrmann, A. Kyrtatos, R. Schulz \& G. Weisser, (2009) "Validation and initial application of a novel spray combustion chamber representative of large two-stroke diesel engine combustion systems", ICLASS.

[10] K., Herrmann, B. Von Rotz, R. Schulz, G. Weisser, B. Schneider \& K. Boulouchos, (2011) "Spray combustion chamber: facility for investigations in relation to large 2-stroke marine Diesel engine combustion optimization", International symposium on marine engineering, Kobe, Japan.

[11] P., Andreadis, A. Zompanakis, C. Chryssakis, \& L. Kaiktsis, (2011) "Effects of the fuel injection parameters on the performance and emissions formation in a large-bore marine diesel engine", Int. J. Engine Res, Vol. 12.

[12] N., Stamoudis, C. Chryssakis \& L. Kaiktsis, (2013) “A two-component heavy fuel oil evaporation model for CFD studies in marine Diesel engines", Fuel.

[13] R.D., Reitz \& R. Diwakar, (1986) “Effect of drop breakup on fuel sprays”, SAE paper,1986. 